\title{
Systemic administration of attenuated Salmonella typhimurium in combination with interleukin-21 for cancer therapy
}

\author{
YUXUAN WANG $^{1}$, JIANXIANG CHEN ${ }^{1}$, BO TANG $^{1,2}$, XIANGYU ZHANG $^{1}$ and ZI-CHUN HUA ${ }^{1,2}$ \\ ${ }^{1}$ The State Key Laboratory of Pharmaceutical Biotechnology, Nanjing University, Nanjing, \\ Jiangsu 210093; ${ }^{2}$ Changzhou High-Tech Research Institute of Nanjing University and \\ Jiangsu TargetPharma Laboratories Inc., Changzhou 213164, P.R. China
}

Received September 29, 2012; Accepted February 19, 2013

DOI: $10.3892 / \mathrm{mco} .2013 .90$

\begin{abstract}
Attenuated Salmonella typhimurium (S. typhimurium) strain VNP20009 has been employed as a powerful anticancer agent due to its selective accumulation in tumors for targeted therapy. S. typhimurium has been demonstrated to constitute a delivery tool carrying antiangiogenic or proapoptotic genes that treat cancer. The hydrodynamic tail vein (HTV) injection of naked plasmid DNA has been developed as an effective gene delivery strategy, which has been successfully used in vivo. The aim of this study was to develop a combination therapy of S. typhimurium VNP20009 and HTV injection of interleukin-21 (IL-21) expression plasmid to evaluate the antitumor potential on an experimental melanoma model. Consistent with previous results, single VNP20009 treatment was demonstrated to possess effective activities to suppress tumor growth and prolong animal survival. Moreover, HTV injection of IL-21 plasmid promoted the antitumor activities of VNP20009. The mice that were administered combined therapy exhibited smaller tumor sizes and longer survival time compared with those administered VNP20009 or IL-21 treatment alone. IL-21 induced more infiltrating natural killer (NK) and T cells to the tumor area. These findings indicated that $S$. typhimurium in combination with IL-21 promotes antitumor immune responses, suggesting a novel strategy in the treatment of cancer.
\end{abstract}

\section{Introduction}

The existence of hypoxic areas is an important parameter for solid tumors, which is due to the excessive consumption of oxygen for tumor growth $(1,2)$. Previous studies have characterized critical resistant roles of tumor hypoxia for radiation

Correspondence to: Professor Zi-Chun Hua, The State Key Laboratory of Pharmaceutical Biotechnology, Nanjing University, No. 22 Hankou Road, Nanjing, Jiangsu 210093, P.R. China E-mail: zchua@nju.edu.cn

Key words: Salmonella typhimurium, interleukin-21, melanoma therapy and most anticancer drugs as the shortage of blood vessels (3). Moreover, extensive research has indicated that tumor hypoxia promotes malignant progression and metastasis (4). Therefore, strategies or drugs targeting hypoxia have become promising in the implementation of tumor-specific treatments.

Attenuated Salmonella typhimurium (S. typhimurium) strains accumulate and grow in tumor environments (5). Therefore, the potential of S. typhimurium in tumor-targeting therapy has been evaluated. S. typhimurium was initially used as a single-agent to treat cancer. Results from prostate cancer, breast cancer and hepatocellular carcinoma demonstrated potential anticancer activities of S.typhimurium (6). Moreover, S. typhimurium is increasingly utilized as a vector to deliver antiangiogenic or proapoptotic genes into the hypoxic area of a tumor (7). We recently showed that the facultative anaerobe S. typhimurium strain VNP20009 is able to replicate in hypoxic tumors and function as a potential vector in an established mouse melanoma tumor model (8-11).

Cytokines have been shown to have significant effects on tumor development. IL-21, one of newly identified cytokines, belongs to a large family of cytokines identified by their receptors which share a common $\gamma$ chain (12). IL-21 is produced by activated $\mathrm{T}$ and natural killer $\mathrm{T}$ (NKT) cells. The major function of IL-21 is to increase the pool of cytotoxic $\mathrm{CD}^{+} \mathrm{T}$, natural killer (NK) and NKT cells (13). Therefore, it is not unexpected that IL-21 has antitumor activity. IL-21 has been proven to possess anticancer activities in various tumors such as melanoma, colon and pancreatic carcinoma (13). Based on this, IL-21 has been used in phase I clinical trials in advanced-stage melanoma patients (13). Thus, IL-21 is becoming a promising cytokine in antitumor research.

In this study, we applied a novel strategy to treat melanoma with S. typhimurium VNP20009 in combination with IL-21 gene therapy, which was delivered by hydrodynamic tail vein (HTV) injection. The combination increased the inhibitory effects on tumor growth compared with S. typhimurium or IL-21 treatment alone, and improved the survival time of animals. More infiltrating NK and T cells were observed in the tumor area of the mice that were administered VNP20009 in combination with IL-21 compared with S. typhimurium VNP20009 treatment alone. These data suggest a novel strategy that can be employed to treat melanoma in vivo. 


\section{Materials and methods}

Plasmid construction. The full-length coding sequence of mouse IL-21 was amplified from the mouse T cell cDNA library and inserted into a vector pBLAST42-mcs (Invivogen, Inc., San Diego, CA, USA). The coding sequence and open reading frame of positive clones were confirmed to be correct by DNA sequencing (GenScript Corporation, Nanjing, China).

Animals. Six- to seven-week-old female C57BL/6 mice were purchased from the Model Animal Research Center of Nanjing University (Nanjing, China) and housed in a specific pathogen-free (SPF) animal facility (Gulou Hospital, Nanjing, China) which was environmentally controlled $\left(22^{\circ} \mathrm{C}\right.$; with a 12:12 h light/dark cycle; light cycle, 08:00-20:00; dark cycle 20:00-08:00) with ad libitum access to standard laboratory chow and water. The protocols were approved by the local Institution Review Boards and the animal experiments were performed in accordance with the Ethical Guidelines for Animal Use and Care established by the Nanjing University (Nanjing, China).

Cell culture and tumor model. B16F10 melanoma cells were purchased from the American Type Culture Collection (ATCC; Manassas, VA, USA) and cultured in Dulbecco's modified Eagle's medium (DMEM; Invitrogen, Carlsbad, CA, USA) with $10 \%$ fetal bovine serum (FBS; Invitrogen). C57BL/6 mice were inoculated subcutaneously (s.c.) on the mid-right flank with $5 \times 10^{5} \mathrm{~B} 16 \mathrm{~F} 10$ cells in $0.1 \mathrm{ml}$ phosphate-buffered saline (PBS). S. typhimurium strain VNP20009 (ATCC) was cultured and prepared as described and then injected intraperitoneally (i.p.) with $0.2 \mathrm{ml}$ PBS containing $1 \times 10^{6}$ colony-forming units (cfu) bacteria into the tumor-bearing mice 6 days post-inoculation. IL-21 expression or control plasmid was injected through the hydrodynamic tail vein (HTV) twice at 6 and 13 days following inoculation. The tumor volume was measured individually with a caliper and determined by the formula: tumor volume $=$ length $\mathrm{x}$ width $\mathrm{x}$ width $\mathrm{x} 0.52$.

Transfection and western blot analysis. 293T cells were cultured in DMEM with 10\% FBS. IL-21 expression or control plasmid was transfected by Lipofectamine 2000 (Invitrogen) following the manufacturer's instructions. Following transfection $(24 \mathrm{~h})$, the cells were collected and homogenized in lysis buffer containing $50 \mathrm{mM}$ Tris- $\mathrm{HCl}(\mathrm{pH} 7.4), 250 \mathrm{mM}$ $\mathrm{NaCl}, 0.5 \%$ Triton X-100, $50 \mathrm{mM} \mathrm{NaF}, 2 \mathrm{mM}$ EDTA and $1 \mathrm{mM} \mathrm{Na} \mathrm{VO}_{4}$ on ice for $30 \mathrm{~min}$. Anti-IL-21 antibody was purchased from R\&D Systems, Inc. (Minneapolis, MN, USA).

Enzyme-linked immunosorbent assay (ELISA). Serum samples were prepared following standard protocol. Samples were assayed using the an ELISA kit (eBioscience, Inc., San Diego, CA, USA). The reagents used were supplied in the kit. Each ELISA plate was read in a Bio-Tex ELx800 ${ }^{\mathrm{TM}}$ plate reader (Cole-Palmer, Vernon Hills, IL, USA). Reader software was used to calculate the data.

Flow cytometric analysis. Single-cell suspension was prepared from the tumor tissues. The suspension was blocked with $10 \%$ FBS for $30 \mathrm{~min}$. Immunofluorescent staining of the cell surface markers was performed using PE-Cy ${ }^{\mathrm{TM}} 5$ rat anti-mouse CD4 for the CD4 T cells, FITC rat anti-mouse CD8 $\alpha$ for the CD8 T cells, and APC mouse anti-mouse NK-1.1 for the NK cells (Becton-Dickinson, Franklin Lakes, NJ, USA). Samples were incubated in the dark by agitation for 45 min. Following washing in PBS, the cells were resuspended and then analyzed.

Statistical analysis. Data were expressed as the mean \pm standard deviation (SD) and data analysis was performed using SPSS software (SPSS 17.0, Chicago, IL, USA). Paired Student's $\mathrm{t}$-test analysis was conducted to assess statistical significance. $\mathrm{P}<0.05$ was considered to indicate a statistically significant difference.

\section{Results}

Effective expression of IL-21 plasmid in vivo. To perform the HTV injection of IL-21 plasmid, we firstly constructed the IL-21 expressing plasmid. The full-length cDNA of the IL-21 gene was amplified from a cDNA library of mouse $\mathrm{T}$ cells with specific primers (Fig. 1A). The positive clones containing IL-21 gene were identified by enzyme digestion and then confirmed by DNA sequencing. The plasmid was then transfected into 293T cells in order to evaluate the expression efficiency of IL-21 protein. The plasmid containing IL-21 cDNA successfully expressed IL-21 in 293T cells, compared with the vehicle control (Fig. 1B). The plasmid was then delivered into mice by HTV injection. Higher IL-21 expression in the serum of mice that received IL-21 expression plasmid was detected using ELISA compared with that of the control (Fig. 1C).

Combination of S. typhimurium and IL-21 inhibits tumor growth and animal survival. Once the IL-21 expressing plasmid was constructed, the antitumor efficiency of $S$. typhimurium VNP20009 combined with IL-21 was examined in vivo. Six days following the establishment of the B16F10 melanoma mouse model, VNP20009 and IL-21 plasmids were delivered by i.p. and HTV injection, respectively. The tumor volumes were monitored from 6 to 28 days. The tumor that received combination treatment exhibited smaller size compared with single treatment controls (Fig. 2A). We found that the combination strategy significantly inhibited the tumor volumes of melanoma compared with that of VNP20009 or IL-21 treatment alone $(\mathrm{P}<0.01$, Fig. 2B). Compared with vehicle controls, the single treatment of VNP20009 and IL-21 prolonged the survival of the mice bearing the melanoma. Notably, the combination strategy significantly prolonged the survival time (Fig. 2C).

IL-21 promotes $T$ and $N K$ cell infiltration. IL-21 has been shown to effectively induce the infiltration of $\mathrm{T}$ and NK cells. Therefore, we investigated whether or not it improved the treatment efficiency through promoting $\mathrm{T}$ and NK cell infiltration in the tumor microenvironment. To investigate this, we examined the T-helper cell distribution in tumors by their specific marker CD4. As expected, IL-21 induced CD4 ${ }^{+} \mathrm{T}$ cell infiltration in tumor $\sim 2$ times compared with controls (Fig. 3A). Similarly, more cytotoxic T cells marked by CD8 were also observed in tumors treated with IL-21 (Fig. 3B). Furthermore, IL-21 induced more NK1.1 cell distribution in tumors (Fig. 3C). These data suggest that IL-21 facilitates the 
A

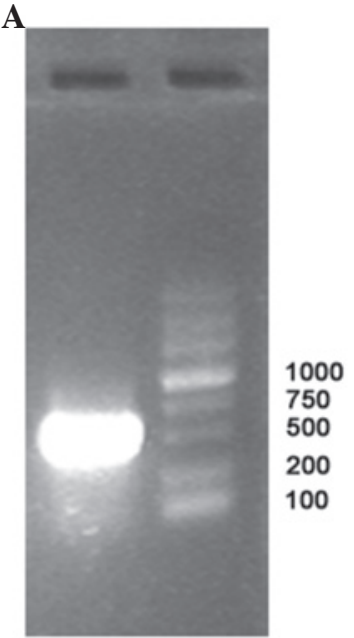

B

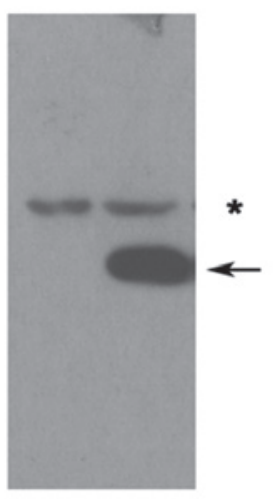

C

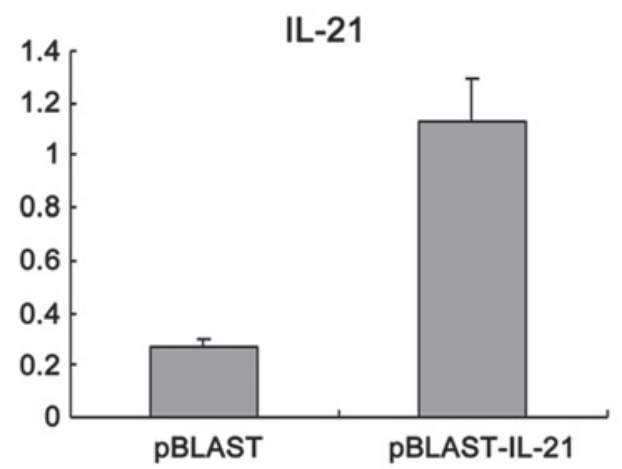

Figure 1. Expression of IL-21 in vivo. (A) Amplification of murine IL-21 from mouse lymphocyte cDNA library. (B) Western blot analysis of IL-21 expression in 293T cells. Asterisk, unspecific bands; arrow, specific band for IL-21. (C) ELISA analysis of serum IL-21 expression from mice undergoing hydrodynamic tail vein injection of IL-21 plasmid. IL-21, interleukin-21.

A

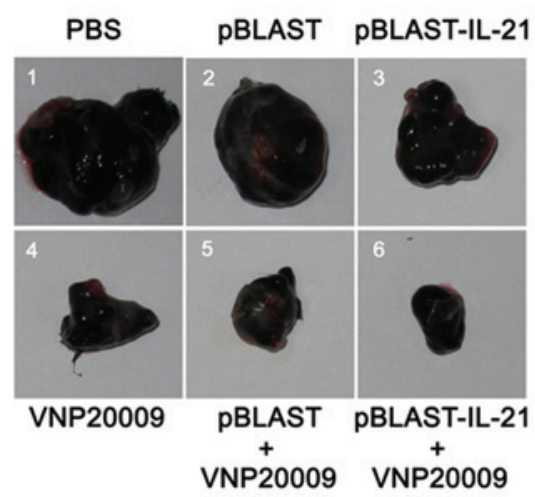

B

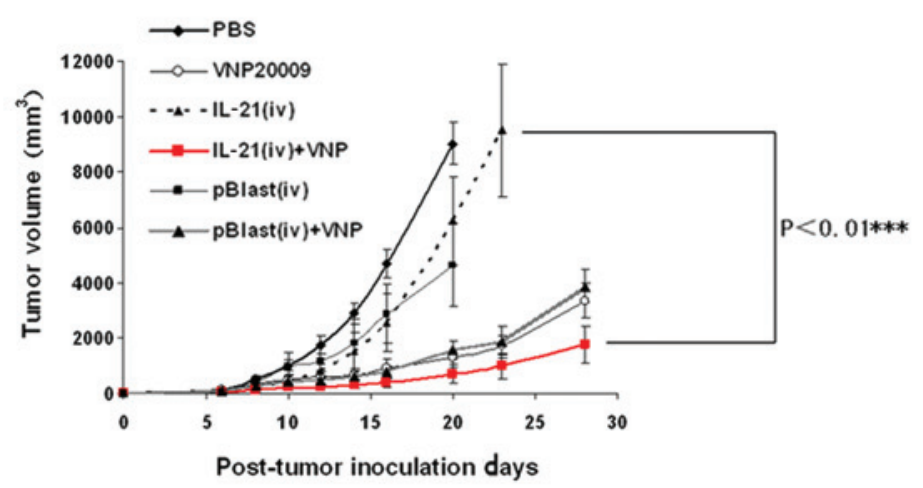

C

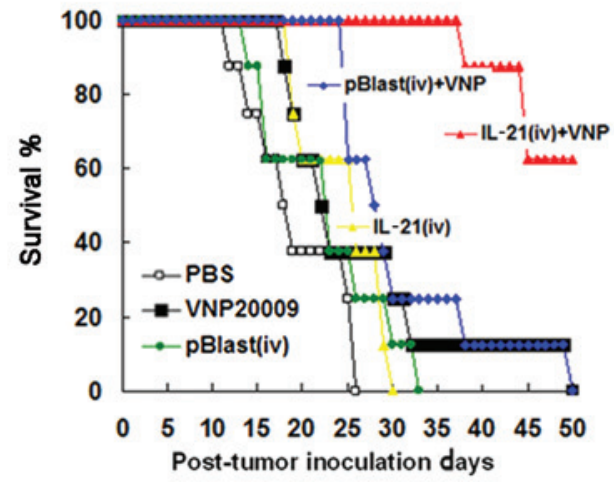

Figure 2. Combination of S. typhimurium and IL-21 inhibits tumor growth and animal survival. (A) Representative melanoma tumor size following treatment as indicated. (B) Effect of the treatment as indicated on the melanoma tumor volumes. (C) Cumulative survival (Kaplan-Meier) of mice treated as indicated. PBS, phosphate-buffered saline; IL-21, interleukin-21.

antitumor activities of VNP20009 by inducing the infiltrated T and NK cells.

\section{Discussion}

Conventional anticancer therapies such as chemotherapy and radiotherapy have successfully been used in treating different types of cancer. However, approximately half of the patients exhibited resistance to these therapies (14). This encourages the development of novel therapies with higher specificity and efficiency. One of the most prevalent differences of tumors from normal tissues is the existence of the hypoxia region. This characteristic is becoming a candidate for novel tumor-targeted therapies. Among many emerging novel 
A

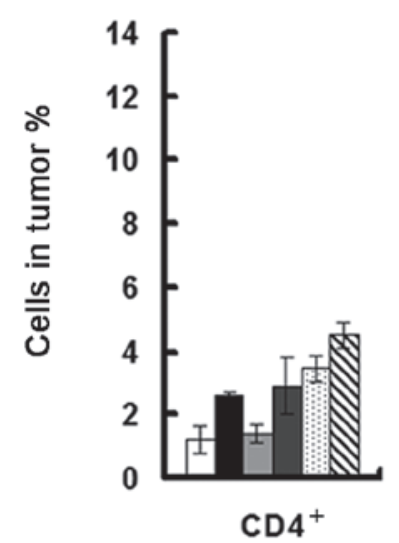

$\square$ PBS

- VNP20009

apblast

a pblast+VNP20009

$\square \mathrm{pmIL-21}$

spmIL-21+VNP20009

C

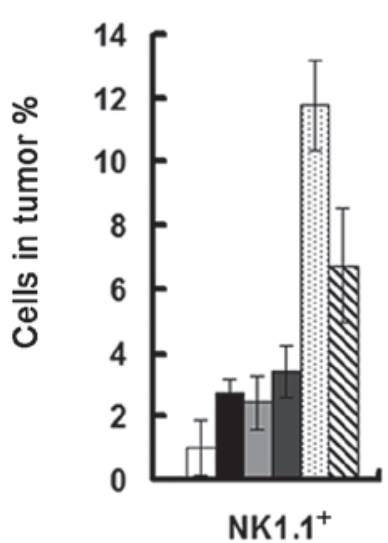

B

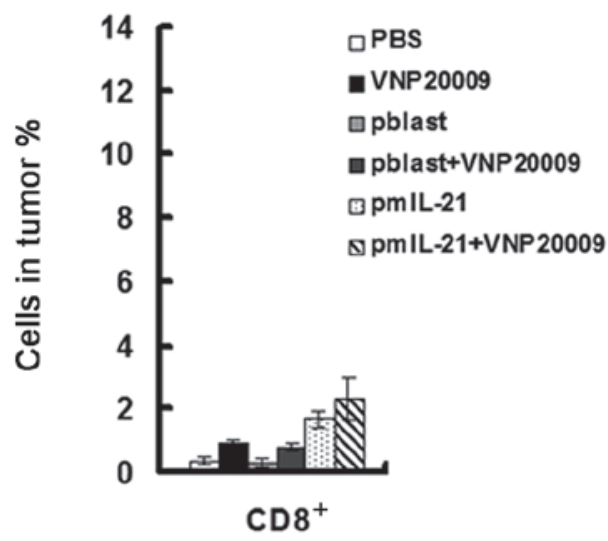

Figure 3. (A) Infiltration of immune cells in the tumor and (B) flow cytometric analysis of CD4 $4^{+}$and $\mathrm{CD} 8^{+} \mathrm{T}$ cells in the melanoma tumor treated as indicated. (C) NK1.1 $1^{+}$cell distribution in the melanoma tumor. PBS, phosphate-buffered saline; IL-21, interleukin-21.

anticancer experimental strategies targeting the hypoxic area, bacterial treatment possesses certain advantages that render it a promising candidate for novel cancer therapies (15). For example, some anaerobic bacteria such as Salmonella, which preferentially replicate in hypoxic and necrotic areas, have been extensively used in the development of novel cancer treatment strategies. To reduce the side-effects of Salmonella as a bacterium, attenuated S.typhimurium have been developed and approved for clinical trials in cancer treatment (16). It has been shown that distinct strategies was developed for Salmonella typhimurium to treat cancer (17). The primary application of $S$. typhimurium in cancer treatment is to directly inhibit tumor growth. Current research combines $S$. typhimurium with other antitumor agents to develop novel strategies. In the present study, we have developed a novel strategy to treat melanoma by combining IL-21 with $S$. typhimurium VNP20009. IL-21 significantly improved the inhibitory effect on the tumor growth of S.typhimurium. More $\mathrm{CD}^{+}$and $\mathrm{CD} 8^{+}$lymphocytes and NK were observed in the tumor after IL-21 administration, indicating that IL-21 is able to effectively promote immune cell infiltration in melanoma tumors. As a newly identified interleukin, the regulatory roles of IL-21 in cancer inhibition remain controversial (18). It has been proven to exhibit inhibitory effects on tumors such as pancreatic carcinoma, fibrosarcoma and renal cell carcinoma. However, it has also been shown to possess accelerative roles in colitis-associated colon cancer $(19,20)$. Therefore, IL-21 has been suggested to have a cell context-dependent activity on cancer. Thus, IL-21 exhibits anticancer effects on melanoma in vivo.

HTV delivery of naked DNA or RNA is a simple and effective in vivo gene delivery method (21). This non-viral strategy has been successfully used to express exogenous genes in tissues such as skeletal muscle and liver and has been successfully used in several clinical trials $(22,23)$. Whether this systematic delivery strategy is useful to cancer treatment has not been fully elucidated yet. In the present study, we showed that HTV injection is an effective strategy to deliver an anticancer gene in vivo, suggesting a novel combination strategy of bacteria and non-viral delivery method to treat cancer. Additional studies are required to evaluate the safety and efficiency of this novel strategy.

\section{Acknowledgements}

The authors are grateful to grants from the National Key Basic Research Program from the Ministry of Science and Technology (2012CB967004), the Jiangsu Provincial Nature Science Foundation (BK2011228, BZ2011048, BZ2010074, BZ2012050), the Chinese National Nature Sciences Foundation (81121062, 50973046, 31071196, 31070706) and the Bureau of Science and Technology of Changzhou (CN20100016, CZ20100008, CJ20115006, CE20115034, CZ20110028). 


\section{References}

1. Dachs GU, Patterson AV, Firth JD, et al: Targeting gene expression to hypoxic tumor cells. Nat Med 3: 515-520, 1997.

2. Pouyssegur J, Dayan F and Mazure NM: Hypoxia signalling in cancer and approaches to enforce tumour regression. Nature 441 437-443, 2006

3. Brown JM and Giaccia AJ: The unique physiology of solid tumors: opportunities (and problems) for cancer therapy. Cancer Res 58: 1408-1416, 1998.

4. Graham CH, Forsdike J, Fitzgerald CJ and MacDonaldGoodfellow S: Hypoxia-mediated stimulation of carcinoma cell invasiveness via upregulation of urokinase receptor expression. Int J Cancer 80: 617-623, 1999.

5. Leschner S and Weiss S: Salmonella-allies in the fight against cancer. J Mol Med (Berl) 88: 763-773, 2010.

6. Luo X, Li Z, Lin S, et al: Antitumor effect of VNP20009, an attenuated Salmonella, in murine tumor models. Oncol Res 12 501-508, 2001

7. Pawelek JM, Low KB and Bermudes D: Tumor-targeted Salmonella as a novel anticancer vector. Cancer Res 57: 4537-4544, 1997.

8. Chen J, Wei D, Zhuang H, et al: Proteomic screening of anaerobically regulated promoters from Salmonella and its antitumor applications. Mol Cell Proteomics 10: M111.009399, 2011.

9. Jia LJ, Wei DP, Sun QM, Jin GH, et al: Tumor-targeting Salmonella typhimurium improves cyclophosphamide chemotherapy at maximum tolerated dose and low-dose metronomic regimens in a murine melanoma model. Int J Cancer 121: 666-674, 2007.

10. Chen G, Tang B, Yang BY, Chen JX, et al: Tumor-targeting Salmonella typhimurium, a natural tool for activation of prodrug $6 \mathrm{MePdR}$ and their combination therapy in murine melanoma model. Appl Microbiol Biotechnol: Aug 7, 2012 (Epub ahead of print).

11. Chen J, Yang B, Cheng X, et al: Salmonella-mediated tumortargeting TRAIL gene therapy significantly suppresses melanoma growth in mouse model. Cancer Sci 103: 325-333, 2012.
12. Spolski R and Leonard WJ: Interleukin-21: basic biology and implications for cancer and autoimmunity. Annu Rev Immunol 26: 57-79, 2008.

13. Sondergaard H and Skak K: IL-21: roles in immunopathology and cancer therapy. Tissue Antigens 74: 467-479, 2009.

14. Goluboff ET, Hirano D, Thrasher JB, Stark G, Miller GJ and Glode LM: New approaches to the treatment of advanced prostate cancer. Rev Urol 3: S69-S78, 2001.

15. Patyar S, Joshi R, Byrav DS, Prakash A, Medhi B and Das BK: Bacteria in cancer therapy: a novel experimental strategy. J Biomed Sci 17: 21, 2010.

16. Low KB, Ittensohn M, Luo X, et al: Construction of VNP20009: a novel, genetically stable antibiotic-sensitive strain of tumortargeting Salmonella for parenteral administration in humans. Methods Mol Med 90: 47-60, 2004.

17. Dang LH, Bettegowda C, Huso DL, Kinzler KW and Vogelstein B: Combination bacteriolytic therapy for the treatment of experimental tumors. Proc Natl Acad Sci USA 98: 15155-15160, 2001.

18. Stolfi C, Pallone F, Macdonald TT and Monteleone G: Interleukin-21 in cancer immunotherapy: friend or foe? Oncoimmunology 1: 351-354, 2012.

19. Stolfi C, Rizzo A, Franze E, et al: Involvement of interleukin-21 in the regulation of colitis-associated colon cancer. J Exp Med 208: 2279-2290, 2011

20. Jauch D, Martin M, Schiechl G, et al: Interleukin 21 controls tumour growth and tumour immunosurveillance in colitisassociated tumorigenesis in mice. Gut 60: 1678-1686, 2011.

21. Hodges BL and Scheule RK: Hydrodynamic delivery of DNA. Expert Opin Biol Ther 3: 911-918, 2003.

22. Romero NB, Benveniste O, Payan C, et al: Current protocol of a research phase I clinical trial of full-length dystrophin plasmid DNA in Duchenne/Becker muscular dystrophies. Part II: clinical protocol. Neuromuscul Disord 12: S45-S48, 2002.

23. Budker V, Zhang G, Knechtle S and Wolff JA: Naked DNA delivered intraportally expresses efficiently in hepatocytes. Gene Ther 3: 593-598, 1996. 